Dossier: El Quehacer de las Humanidades: Acción Social, Investigación y Metodologías e Innovación Tecnológica en la Docencia

\title{
Pertinencia de la Guía de Investigación del Curso Integrado de Humanidades de la Escuela de Estudios Generales de la Universidad de Costa Rica. Una perspectiva crítica: autocomplacencia o autocrítica
}

\author{
Vanessa Montalbán Rivera \\ Universidad de Costa Rica, Costa Rica \\ vanessa.montalban@ucr.ac.cr \\ https://orcid.org/0000-0003-2265-485X
}

Recibido: 18 de octubre de 2019

Aceptado: 15 de enero de 2020

Resumen: En la Universidad de Costa Rica, en el Sistema de Educación General, en la Escuela de Estudios Generales, se imparte un curso inter y transdisciplinar, que nace con la intención de brindar un espacio de formación humanista; integral e integrador de la persona y del futuro profesional, que le introduce en el quehacer universitario desde lo académico y cultural. Es el "Curso integrado de Humanidades".

Una parte de este en el subcurso denominado: "Guía Integrada de Investigación", que inicia a los estudiantes en los caminos de la investigación. Este es el objeto del presente artículo, cuya pregunta fundamental versa sobre su pertinencia, puesto que por las complejidades que conlleva se ha cuestionado su existencia y forma de realizarse.

Se mostrará una posible respuesta con fundamentación teórica e ideológica, desde la normativa de la Universidad de Costa Rica, el ideario de los Estudios Generales, la experiencia personal y docente.

\section{(c) (i) (9) (2)}

La Revista Estudios es editada por la Universidad de Costa Rica y se distribuye bajo una Licencia Creative Commons Atribución-NoComercial-CompartirIgual 3.0 Costa Rica. Para más información envíe un mensaje a 
Especial: Profesores de Estudios Generales investigan

Palabras clave: Guía Académica; metodologías de investigación; docencia universitaria; Humanidades.

\title{
Pertinence of the Guía Académica in the Curso Integrado de Humanidades at the Escuela de Estudios Generales at the Universidad de Costa Rica: a Critical Perspective: Complacency or Self-Criticism
}

\begin{abstract}
At the Universidad de Costa Rica, part of the Sistema de Educación General, in the Escuela de Estudios Generales, there's an inter and transdisciplinar course called Curso Integrado de Humanidades. This course has the intention of giving humanistic and integral formation to the person and future UCR's professional because it gives the basics academic and cultural university's labour. In this course, there is a sub-course called "Guía Integrada de Investigación" which has the objective of teaching the basics of academic research. The objective of this paper is questioning the pertinence of this program because, during the years, its complexity has arisen this idea: not only for its existence but also for the way it is taught. This paper shows a possible theorical and ideological answer, taking into consideration the law that orders the UCR, the ideals of the Escuela de Estudios Generales, and the personal teaching experience.
\end{abstract}

Keywords: Guía Académica; research methodologies; teaching experience; university teaching; Humanities.

\section{Presentación}

La Universidad de Costa Rica (UCR) es la más antigua institución de educación superior en el país, cuya apertura se da en 1941; sus antecedentes históricos se remontan a la Casa de Enseñanza de Santo Tomás, en 1824 y posteriormente a la Universidad de Santo Tomás en 1843, clausurada en 1888. Tiene una larga trayectoria de aportes a la comunidad nacional e internacional, en las áreas de la docencia, la investigación y la acción social. Es una institución

\section{(c) (i) (2)}

La Revista Estudios es editada por la Universidad de Costa Rica y se distribuye bajo una Licencia Creative Commons Atribución-NoComercial-CompartirIgual 3.0 Costa Rica. Para más información envíe un mensaje a 
ISSN 1659-3316

Montalbán Rivera Vanessa

Especial: Profesores de Estudios Generales investigan

relevante para la comunidad nacional, que constitucionalmente ha garantizado su funcionamiento, autonomía y que también estableció el mandato constitucional para el Estado y en particular para el órgano legislativo, de escuchar sus criterios sobre los proyectos de ley que estén bajo sus competencias. Este contexto anuncia ya la responsabilidad de brindar un conocimiento crítico y riguroso sobre los asuntos trascendentes de la vida nacional.

En la Universidad de Costa Rica hay unos procesos históricos de reflexión y revisión, que a lo largo de los años han venido perfilando su identidad y quehacer; son los Congresos Universitarios. En 1957, como fruto de uno de estos Congresos, se crea la Facultad Central de Ciencias y Letras de la Universidad de Costa Rica y en ella se cuenta con el Departamento de Estudios Generales. Este responde a la necesidad que vio un grupo distinguido de docentes de la época, que ante el vertiginosos desarrollo de la ciencia y la tecnología, se preocupaban porque la formación de los futuros profesionales se limitara solamente a la especialización (Láscaris, 1958, p.4), olvidando la formación cultural y humana en general, por lo que crean unos cursos de estudios generales; que serán albergados primero en el Departamento y después en la Escuela de Estudios Generales (EGG), que a su vez serán incorporados al Sistema de Educación General (SEG) que se crea posteriormente en 1973. Estos cursos tenían la intención de llenar ese vacío formativo, se convertirían a lo largo de los años, en el sello distintivo de esta casa de enseñanza y de sus profesionales; pues en ellos se concretiza el ideal de formación humanista que se propuso en la época, tal y como lo dijo el profesor José Joaquín Trejos, en su discurso inaugural de la Facultad de Ciencias y Letras en 1957:

Los Estudios Generales deben dejar... un conjunto de conocimientos; pero, muy principalmente deben dejar también, como residuo, un semillero de intereses e inquietudes intelectuales y aprecio de los mejores valores éticos y artísticos que ha producido la humanidad, capaz de perdurar y fructificar en sus espíritus a través de sus vidas ... (Láscaris, 1958, p. 30).

\section{(c) (i) (2)}

La Revista Estudios es editada por la Universidad de Costa Rica y se distribuye bajo una Licencia Creative Commons Atribución-NoComercial-CompartirIgual 3.0 Costa Rica. Para más información envíe un mensaje a 
Especial: Profesores de Estudios Generales investigan

La conformación del "Curso integrado de Humanidades" o "Humanidades" o "Generales" como se le suele llamar, a lo largo del tiempo ha ido incorporando modalidades metodológicas y diversas experiencias docentes tendientes a cumplir estas expectativas. Fundamentalmente es una actividad pedagógica interdisciplinaria, realizada por un grupo docente especializado en Filosofía, Historia, Castellano y Literatura que posteriormente será Comunicación y Lenguaje. Disciplinas, conocimientos y quehaceres que a su vez conforman la base del curso integrado, que se consideran necesarias, elementales para la formación profesional y humana que se busca. Se le incorporan además otras disciplinas de las ciencias sociales, "exactas" y artes. Es un curso integrado no sólo desde el punto de vista del trabajo docente, metodológico, sino también evaluativo, que tiende además a la transdisciplinariedad.

En palabras del profesor Isaac Felipe Azofeifa dirigida a los estudiantes de primer ingreso a la Universidad, se explica mejor su propósito: "El plan propuesto buscará siempre logar que cada uno de ustedes integre su cultura con una visión de los campos del conocimiento distintos de los que tendrán obligatoriamente que dominar en el curso de su especialización profesional” (1979, p.72).

El curso se lleva a la práctica a través de dos modalidades pedagógicas: la opción llamada "Regular", conformada por tres subcursos disciplinares; Filosofía, Historia, Comunicación y Lenguaje, cada una a cargo de un docente especialista. Se le suma un cuarto subcurso llamado "Guía Integrada” o "Guía Académica", un curso cuya labor de introducción a la investigación, es llevada a cabo como una actividad conjunta, transdisciplinar, por los tres docentes.

La otra opción metodológica para el "Curso Integrado de Humanidades", es la llamada "Seminarios Participativos". En esta modalidad se cuenta con tres docentes de las especialidades base: Filosofía, Historia, Comunicación y Lenguaje, a los que se suma un cuarto docente de una especialidad ya sea de las ciencias sociales, "exactas" o de artes. En este caso, es un solo curso sin

\section{(c) (i) (2)}

La Revista Estudios es editada por la Universidad de Costa Rica y se distribuye bajo una Licencia Creative Commons Atribución-NoComercial-CompartirIgual 3.0 Costa Rica. Para más información envíe un mensaje a 
Especial: Profesores de Estudios Generales investigan

subcursos disciplinares, con un grupo de cuatro docentes que realizan una actividad conjunta integrada, inter y transdiciplinar. No existe, como en el caso de la anterior, un subcurso para la "Guía Integrada", sin embargo como en la otra opción metodológica, se procuran incorporar procesos introductorios de investigación (Espinosa, 2013, p. 139).

El caso particular de la llamada "Guía Integrada" de la opción metodológica "Regular", es de la que se ocupa el presente artículo, pues al funcionar como un cuarto subcurso en el que se debe integrar el trabajo conjunto de los especialistas, conlleva una serie de retos que si bien no están ausentes en la otra opción metodológica, por la modalidad de trabajo que conlleva en la opción "Regular", permite que se contemplen más claramente, de tal forma que su reflexión también será un aporte para el quehacer de las dos opciones metodológicas.

\section{Autocomplacencia o autocrítica}

La reflexión y análisis que se propone en este artículo, debe empezar por la síntesis de los argumentos de los docentes sobre el subcurso de "Guía Integrada" de la opción metodológica "Regular" y de lo que sucede en la práctica. Lo que no quiere decir que haya desacuerdo en cuanto a la importancia de la investigación como tal, del valor que tiene por sí misma y de la relevancia en la formación de los profesionales de la UCR y en su quehacer en general. Lo que se presenta a continuación es el recuento de conversaciones recientes en las que quien escribe quería conocer expresamente, los análisis del personal docente que imparte el curso, a raíz de la elaboración de una ponencia que presentaría en el pasado Coloquio sobre las Humanidades, realizado del 6 al 8 de agosto del 2019. Es también el recuento de experiencias y comentarios recogidos a lo largo de más de 15 años de práctica docente en estos cursos. 
Especial: Profesores de Estudios Generales investigan

\section{Entonces, ¿dónde estaban y están los peros sobre la "Guía Integrada}

de Investigación"? Algunos docentes consideran que el problema ha estado en la forma de organizar la actividad de la "Guía Integrada de Investigación", en la oferta educativa de la Escuela de Estudios Generales, en la del "Curso Integrado de Humanidades", en que no hay trabajo en equipo, en que la estructura administrativa no propicia la consolidación de los grupos de trabajo de los docentes. También, en que se deberían ofrecer otras opciones, ya que hay muchas maneras de investigar, en que los procedimientos son "cuadrados", poco flexibles, en que se pueden proponer ensayos que no requieren la formulación de problemas y objetivos. Además, alegan que hay otros estilos o tipos de investigación de otra diversidad de disciplinas, que por lo mismo se atenta contra la creatividad. Por otro lado, con respecto a la dinámica de trabajo que a veces es interpretada por algunos docentes, como que la "Guía Integrada de Investigación" se trata de guiar a "mis grupos" y que en las presentaciones finales son "mis grupos" contra "tus grupos", de tal forma que se pierde de vista la finalidad de la dinámica y se cae en una especie de reto o competencia entre colegas, que puede llevar hasta a venganzas o "sacadas de clavo", o hasta simplemente ignorar la nota colegiada que conlleva y beneficiar a unos estudiantes con una nota desproporcionada y dejar en desventaja a otro grupo de estudiantes que fueron evaluados según las regulaciones vigentes. En el caso de algunos docentes, se le valora como una actividad irrelevante, que simplemente debe ser cumplida y en este caso da lo mismo que los estudiantes tengan un 10 de nota final.

Otras inquietudes propias de la práctica son y eran: ¿Cómo será pertinente el trabajo en equipo cuando no somos un equipo que nos formamos ni planificamos juntos? ¡Y les pedimos eso a los estudiantes!; no existe la posibilidad de elegir un equipo de trabajo; hay un programa que es inamovible, que no se revisa y luego la lotería de con quién nos corresponderá trabajar; terminamos haciendo una chabacanería. ¿Será que debe convertirse en un curso específico

\section{(c) (i) (2)}

La Revista Estudios es editada por la Universidad de Costa Rica y se distribuye bajo una Licencia Creative Commons Atribución-NoComercial-CompartirIgual 3.0 Costa Rica. Para más información envíe un mensaje a revistaestudios.eeg@ucr.ac.cr. 


\section{Especial: Profesores de Estudios Generales investigan}

que pueda impartir cualquier docente? ¿Hay voluntad para el cambio? ¿Una oferta académica distinta para abordar la investigación en Estudios Generales?

Frente a estas realidades conocidas a lo largo de los años, en distintos contextos y grupos de trabajo, es importante preguntarse: ¿tiene sentido el subcurso de la "Guía Integrada de Investigación?, ¿es pertinente?, ¿corresponde a los ideales que dieron origen a los Estudios Generales?, ¿viene a propósito de la formación Humanista?, ¿es una actividad que deberíamos seguir haciendo?

Primero hay que advertir que las dificultades o debilidades que se enfrentan al realizar esta actividad, que ciertamente son reales, podrían hacer perder de vista la actividad como tal y por eso se podría explicar un cierto desaliento que se permea en el recuento anterior y que es comprensible plenamente. Sin embargo, podría llevar a renunciar a una actividad que no necesariamente ha perdido vigencia, así como su valor. Asunto que en todo caso es objeto de esta reflexión. En segundo lugar, se podría perder de vista la cuestión sobre los inconvenientes que este curso conlleva, que a lo mejor son el objetivo real, es lo que se debe analizar para disolver, desparecer o resolver lo relativo a la "Guía Integrada de Investigación".

Utilizando la metáfora del Dr. Leonardo Sancho en su ponencia de cierre al citado Coloquio de Humanidades, al respecto del naufragio y los desplazamientos, ¿Cuáles son esos posibles riscos, corales, formaciones rocosas que nos amenazan con un naufragio?, con la posible desaparición del curso de la "Guía Integrada de Investigación":

Considerando lo dicho, concretamente esos riscos podrían ser:

1. La estructuración del curso como tal, sintetizado en los elementos que se recogen en un programa de curso: justificación, descripción, objetivos, contendidos, metodología, cronograma y fundamentación.

2. La sistematización de la práctica de dicha actividad: estrategias didácticas, integración del personal docente. 
Especial: Profesores de Estudios Generales investigan

3. La estructura institucional para la realización de dicha actividad.

¿Cuáles son los nortes que pueden orientar este desplazamiento, legitimar la reflexión y llevarnos a soluciones?, es decir a evitar el naufragio y orientar el desplazamiento hacia horizontes prometedores. Sin olvidar el diálogo intergeneracional e interdisciplinar. A continuación, una reflexión desde los riscos.

\section{Ubicar el contexto}

El curso se imparte en una universidad pública, en la que el docente se debe ubicar como funcionario público y que tiene una larga trayectoria docente que ha heredado. Institución con unas características específicas y distintivas: la de la formación humanista, transversalizada por la docencia, la investigación y la acción social. Supuestos que podrían parecen remotos para el asunto concreto del que se ocupa en este artículo, pero que tienen que ver con el hecho de preguntarse si el curso de la "Guía Integrada de Investigación" debe seguir existiendo, si es pertinente siquiera la pregunta. Posteriormente, tiene que ver con la misma revisión de lo que se hace o debe hacerse en el curso.

Entonces: ¿cuáles son los deberes que se desprenden de ese contexto específico?, ¿cuáles son los recursos con los que se cuenta?, ¿qué dice el Estatuto Orgánico?, ¿otras normativas? y ¿la Constitución Política?, ¿qué dice la normativa y la memoria de los fundamentos de la creación de la Escuela de Estudios Generales? Esto último es fundamental, pues se debe recordar que el docente de estos cursos, ha recibido una herencia a potenciar. Porque "quien no recuerda de dónde viene, no sabe a dónde va", según decía uno de los ponentes del mencionado Coloquio.

Según la Constitución Política de la República de Costa Rica, en su artículo 11, reformado en el año 2000:

\section{(c) (i) (2)}

La Revista Estudios es editada por la Universidad de Costa Rica y se distribuye bajo una Licencia Creative Commons Atribución-NoComercial-CompartirIgual 3.0 Costa Rica. Para más información envíe un mensaje a revistaestudios.eeg@ucr.ac.cr. 


\section{Especial: Profesores de Estudios Generales investigan}

Los funcionarios públicos son simples depositarios de la autoridad. Están obligados a cumplir los deberes que la ley les impone y no pueden arrogarse facultades no concedidas en ella. pública.

... La acción para exigirles la responsabilidad penal por sus actos es

La Administración Pública en sentido amplio, estará sometida a un procedimiento de evaluación de resultados y rendición de cuentas, con la consecuente responsabilidad personal para los funcionarios en el cumplimiento de sus deberes. La ley señalará los medios para que este control de resultados y rendición de cuentas opere como un sistema que cubra todas las instituciones públicas.

Puede afirmarse con propiedad que el cuestionamiento sobre el curso de la "Guía Integrada de Investigación", que se ubicaría en el risco que se denominó "estructura institucional", es pertinente y además es una obligación, por cuanto por mandato constitucional, se deben evaluar los resultados y rendir cuentas sobre los quehaceres de la función pública. Esto implicaría preguntarse cuáles y cómo han sido los procesos de evaluación, reflexión y corrección del quehacer de este curso, a lo largo de sus más de cincuenta años de existencia. La historia da cuenta de reflexiones que se han hecho al respecto, sin embargo, la eficacia y eficiencia de esos procesos es un asunto digno de otro artículo y mejor aún de una investigación más profunda. Por ahora valga sintetizar, que la "Guía Integrada de Investigación" inició por ahí de los años sesenta, no como un proceso de investigación, sino como una labor más bien de orientación y acompañamiento académico para los estudiantes.

Uno de los problemas principales que tuvo que afrontar Estudios Generales, era la deficiente preparación básica y general que tenían los estudiantes al ingresar a la Universidad; esto fue objeto de preocupación en 1965 y 1966 cuando se puso énfasis en mejorar la orientación de los estudiantes con base en tres instrumentos: Guía Académica, la Escolaridad y el Examen Comprensivo (Centro de Investigaciones Históricas, 1991, p. 160).

A finales de esa década se propone que sea una actividad obligatoria para los estudiantes. Para los años setenta, la Guía empieza a dar un giro hacia la

La Revista Estudios es editada por la Universidad de Costa Rica y se distribuye bajo una Licencia Creative Commons Atribución-NoComercial-CompartirIgual 3.0 Costa Rica. Para más información envíe un mensaje a revistaestudios.eeg@ucr.ac.cr. 
ISSN 1659-3316

Montalbán Rivera Vanessa

Especial: Profesores de Estudios Generales investigan

investigación, por un lado por el contexto histórico y por otro por la dinámica misma del trabajo del curso: "Los problemas del desarrollo y del subdesarrollo abrieron una nueva veta para temas de investigación que estuvieran más cercanos a la realidad tanto de los docentes como de los educandos" (Chaverri, A., et al., 1994, p. 100). En cuanto a la dinámica propia de esta actividad de orientación, implicaba por ejemplo la elaboración de fichas de lectura, técnica de lectura guiada, la sugerencia de principios metodológicos y técnicas de enseñanza, entre otras cosas. A finales de la década se empieza a perfilar ya la necesidad de técnicas de investigación, para 1976 se enrumba a la actividad que se ha desarrollado desde entonces, como "Guía Integrada de Investigación", perdiendo poco a poco ese perfil inicial de guía de orientación académica (p.101).

Dicho lo anterior en cuanto a la naturaleza de la "Guía Integrada de Investigación" y a la responsabilidad de rendir cuentas, es importante recordar el contexto institucional de esta actividad y reconocer el carácter trascendente que tiene, no sólo el curso como tal, por cuanto es parte de la formación profesional del estudiantado, sino también con respecto a la trascendencia que para la institución conlleva esta actividad, tal y como se ha perfilado en cuanto a proceso de investigación, lo cual es relevante según lo contenido en el Estatuto Orgánico de la Universidad de Costa Rica, en su artículo 1, como institución: "dedicada a la enseñanza, la investigación, la acción social, el estudio, la meditación, la creación artística y la difusión del conocimiento".

En relación con el risco de la estructura institucional, es importante la revisión de esta actividad, no solo como iniciativa propia de los docentes que la llevan a cabo, sino como parte de un proceso ya institucionalizado en el Estatuto Orgánico en su artículo 4:

"b) Excelencia académica e igualdad de oportunidades: Velar por la excelencia académica de los programas que ofrezca,

g) Acción universitaria planificada: Desarrollar una acción universitaria planificada en pro del mejoramiento".

La Revista Estudios es editada por la Universidad de Costa Rica y se distribuye bajo una Licencia Creative Commons Atribución-NoComercial-CompartirIgual 3.0 Costa Rica. Para más información envíe un mensaje a 
Especial: Profesores de Estudios Generales investigan

Con lo cual se debe afirmar que es una obligación evaluar la labor que se realiza en la "Guía Integrada de Investigación", de tal forma que desaparecerla sin una evaluación previa sería una decisión impensable desde la normativa.

\section{Soluciones: trascendencia de lo que hacemos}

Es probable que los vaivenes de una actividad de tantos años, con experiencias y personas diversas, amenace con un naufragio, tal y como podría percibirse si recordamos parte de los comentarios de docentes a lo largo del tiempo. Pero, tal vez, una posible respuesta sea elevar la mirada, como seguramente lo harán los marineros buscando tierra firme en medio de la tormenta, mirando al horizonte en busca de una ruta posible a tierra firme. Es empezar por elevar la mirada de la realidad inmediata, "pequeña" inscrita, en el reducto del aula y tener una mirada trascendente, que vaya más allá, de tal forma que permita dar cuenta del horizonte que se ha perdido de vista.

Esa mirada que, dirigida al horizonte, percibe las dimensiones del viaje, por las que valdría la pena seguir navegando, parte de esto se encuentra nuevamente en el Estatuto Orgánico en su artículo 5, en el que se establecen una serie de principios orientadores, que recuerdan que dentro de las actividades formativas que se llevan a cabo en la Universidad, se debe contribuir a "la formación de una conciencia creativa y crítica", para la comunidad nacional. Lo cual sin duda se logra entre otras cosas, a través de la investigación, pues las técnicas y la introducción que se hace de esto en al curso de la "Guía Integrada de Investigación", permite reconocer la importancia de la búsqueda de fuentes de información con discernimiento, de los fundamentos sobre los cuales se desarrolla un tema, entre otras actividades, que logran una visión inicial de lo que sería un conocimiento fundamentado y con ello un análisis crítico de las cosas. Se espera que esto se desarrolle a lo largo de la formación universitaria y que culmine con el 
Especial: Profesores de Estudios Generales investigan

progreso de la humanidad en sus diferentes manifestaciones, ciencia y tecnología, artes y humanidades. Que deben ser base del conocimiento de la realidad nacional para transformarla. Para que esto sea realidad algún día, es necesario empezar en "pequeño", en una actividad como la que se propone en la "Guía Integrada de Investigación”. El estudio que fundamenta el pensamiento crítico y que permite el cambio para mejor o el bien de la comunidad nacional, que hace posible la existencia de las universidades públicas, requiere de investigación y el inicio está en este curso.

Otro elemento de trascendencia se desprende de la toma de conciencia o la recuperación de ella en cuanto al valor de cada persona, de la responsabilidad que se tiene en la función y el servicio público, que debe ser asumido en las instancias de toma de decisiones. Pues parte de los argumentos de los docentes, conllevan una serie de situaciones que deben ser corregidas institucionalmente y que, aunque trascienden el quehacer directo en el aula, repercuten en él. Tiene que ver con el tema del trabajo en equipo, la falta de consolidación de los equipos de trabajo, los procesos de capacitación, inducción, supervisión, entre otros, que deben ser parte de las políticas de mejoramiento continuo, excelencia académica y en el desempeño. Por ejemplo: La Asamblea Universitaria órgano de más alta jerarquía, la Asamblea Plebiscitaria conformada por los profesores en Régimen Académico, la Asamblea Colegiada Representativa, el Consejo Universitario, los Consejos Coordinadores de Áreas, entre otras instancias universitarias. Podría parecer que no es necesario mencionar este punto, que a lo mejor es tan evidente, sin embargo, lo cierto es que hay una gran ausencia de participación en estos órganos, por cuanto en las postulaciones y elecciones son pocos los que se ofrecen o participan. Es probable que impere como a nivel nacional, la "cultura" del "yo no me meto", pero cada vez que alguien hace eso, deja que se "meta" otro, que es el que decide. Con el agravante que a veces decide sobre procesos o instancias de las que ni siquiera participa o forma parte de ellas.

\section{(c) (i) (-)}

La Revista Estudios es editada por la Universidad de Costa Rica y se distribuye bajo una Licencia Creative Commons Atribución-NoComercial-CompartirIgual 3.0 Costa Rica. Para más información envíe un mensaje a 
ISSN 1659-3316

Montalbán Rivera Vanessa

Especial: Profesores de Estudios Generales investigan

\section{¿Con qué recursos cuenta?}

¿Qué otros elementos adicionales a la mirada trascendente, tiene el docente que navega el curso de la "Guía Integrada de Investigación"? En el Reglamento de Régimen Académico y Servicio Docente, se le garantiza que la Vicerrectoría de Docencia, junto con las unidades académicas, "fortalecerá el desarrollo académico del personal docente" (Art. 19). Es decir, aquellas dificultades que el docente tenga al ejecutar, planificar y desarrollar el curso de la "Guía Integrada de Investigación", podrá subsanarlas entre otras cosas con procesos de formación docente, capacitación, garantizadas por las instancias mencionadas.

Cuenta además con el respaldo de las Políticas de la Universidad de Costa Rica 2016-2020, como se puede ver en el Eje: Universidad y Sociedad, tendiente a afianzar la investigación, fortalecer la docencia, su interrelación e integración, pues junto con la acción social, son consideradas actividades sustantivas. Por los que el docente puede y debe esperar y solicitar el respaldo debido.

Algunos de los comentarios docentes sobre las dificultades del desarrollo de la "Guía Integrada de Investigación", pasan por problemas de gestión: falta de trabajo en equipo y la consolidación de equipos de trabajo, por lo que es oportuno recordar este apartado de la Política y Eje citados:

2.2.2. Ajustará los procesos de gestión institucionales y la normativa universitaria que sea necesaria, para garantizar que se estimule y potencie el trabajo inter-, multi- y transdisciplinario, en los ámbitos de la docencia, la investigación y la acción social en toda la comunidad universitaria.

Se cuenta además con la posibilidad de diseñar e implementar autoevaluaciones, por lo que se puede ir más allá de la autocomplacencia o la 
Especial: Profesores de Estudios Generales investigan

amenaza y dar un paso hacia una verdadera evaluación para la propia mejora continua, tendientes a garantizar y resguardar la excelencia académica, pues recuérdese que el curso de la "Guía Integrada de Investigación", es una iniciativa heredada, que respondía a las necesidades de los tiempos, a la identidad institucional y al ideal de formación humanista.

Las coordenadas del mapa para evitar el naufragio están dadas en los Lineamientos para la Implementación de un Modelo de Gestión de la Calidad en la Universidad de Costa Rica, aprobados en el 2004, algunos de ellos son:

EFICIENCIA: ... formación de profesionales, resultados de investigación y de acción social.

IDONEIDAD: ... capacidad de la institución de cumplir con las tareas específicas que se desprenden de su misión y propósitos.

ÉTICA: El respeto al significado de las normas, a los usuarios, al personal, a las instituciones relacionadas con la organización, a su entorno social, etc., ha de formar parte de su preocupación por el mejoramiento constante. MEJORAMIENTO DE LA CALIDAD: ... conjunto de actividades..., que permita detectar y eliminar sistemáticamente ineficiencias; prevenir problemas

EVALUACION DE LA CALIDAD: ... proceso formativo, participativo, permanente, sistemático, dirigido a recolectar la información, que permite comparar los logros ... y [...] responden a las necesidades de la Institución, de la comunidad, de quienes utilizan los servicios, así como de los funcionarios y las funcionarias que realizan el trabajo.

La normativa y políticas vigentes también prevén una serie de mecanismos que podrían ser parte de las acciones para enrumbar la nave, curso de la "Guía Integrada de Investigación": talleres de inducción, capacitación a los encargados de propiciar la gestión, formación de equipos de trabajo para la reflexión y autoevaluación frecuentes, encuestas frecuentes, documentación de proceso, informes de calidad. Mecanismo que consideran la satisfacción de quienes reciben y prestan este servicio formativo que es la "Guía Integrada de Investigación".

\section{(c) (i) (2)}

La Revista Estudios es editada por la Universidad de Costa Rica y se distribuye bajo una Licencia Creative Commons Atribución-NoComercial-CompartirIgual 3.0 Costa Rica. Para más información envíe un mensaje a 
Especial: Profesores de Estudios Generales investigan

Como conclusión una pregunta que despierta la reflexión crítica que prometía este artículo, la existencia de los tesineros ¿Una instancia que debería de desparecer? o ¿no debió haber existido?

Esta actividad a que alude la pregunta es una iniciativa, llevada adelante por estudiantes de Filología y respaldada por diversas instancias institucionales, tendiente fundamentalmente a asesorar a los estudiantes que cursan la "Guía Integrada de Investigación". Este contexto remite a uno de los subtítulos anteriores: ¿autocrítica? o ¿autocomplacencia?

Si se es autocomplaciente, podría decirse que los tesineros son una actividad complementaria innecesaria, puesto que para eso hay un equipo interdisciplinario docente, que lleva a cabo una buen labor, que hace que los tesineros sean solo un recurso de los estudiantes poco disciplinados, que no cumplen con su trabajo y que encuentran un auxilio en este grupo, que les resuelve la tarea que no hacen en su propio proceso de investigación. Otros dirán que se vuelven una actividad que propicia la confusión y la contradicción para los estudiantes, pues contradice o se aleja de las orientaciones e indicaciones del equipo docente que dirige los grupos estudiantiles de investigación en el curso.

Si se es autocrítico, tal vez deba admitirse con cierto dejo de vergüenza y desilusión, que los tesineros son un mal necesario, nacido de la falta de eficiencia y eficacia de la labor que algunos docentes hacen en el curso de la "Guía Integrada de Investigación". Entonces resuena nuevamente el dicho de los docentes que se contextualizaba al inicio de este artículo y que explican en parte, por qué este curso, a veces no llega a buen puerto. Hay una triple responsabilidad, una que compete a los estudiantes, que cuando no se apropian de su propio proceso de aprendizaje, naufragan en el curso y buscan a los tesineros como salvavidas desesperados. Otro los docentes que por desidia o por desaliento han perdido el rumbo del curso que deben navegar, llevar a buen puerto y terminan encallando, con un grupo de estudiantes sobrevivientes que posiblemente no 
Especial: Profesores de Estudios Generales investigan

aprendieron nada. Por último, una estructura institucional desarticulada, en la que todos hacen, pero nadie supervisa lo que se hace, de tal forma que en este naufragio, solo queda el "sálvense quien pueda".

Sin embargo, por sombrío que parezca el panorama, la realidad es que junto este paisaje de naufragios, hay muchos otros que llegan a buen puerto. Dichosamente la mayoría de los estudiantes se apropian de su propio proceso de aprendizaje: resulta muy inspirador verles llegar a buen puerto al final del semestre con sus proyectos o tesinas desarrollados con gran compromiso de su parte. Es estimulante el trabajo de docentes que responden no solo al esfuerzo de estudiantes como estos, sino que están ya de por sí comprometidos con la responsabilidad formativa que les ha sido encomendada.

Sin duda queda mucho por hacer y la autocrítica pasa por reconocer las ineficiencias, así como por buscar las eficiencias. Quien escribe este artículo termina afirmando que si bien es cierto hay dificultades, riscos que se asoman y amenazan con el naufragio, son muchos más los recursos para llegar a buen puerto e incluso para trazar nuevas rutas y horizontes que planifiquen el valor que tiene la investigación para la formación profesional, personal y para aportar a la comunidad nacional que tanto espera de la formación en educación superior pública. Hay una gran cantidad de estudiantes que justifican con su trabajo, no escatimar en esfuerzos docentes para acompañarlos en un proceso formativo de excelencia; hay docentes comprometidos, que trabajan todos los días con la mejor disposición de colaborar y lograr la verdadera interdisciplinariedad. Hay una serie de recursos institucionales contendidos en la normativa que se convierten en herramientas muy valiosas para la mejora. Llegar a buen puerto en una actividad conjunta, empieza por el compromiso personal, que a juicio de quien escribe, es lo primero que se necesita para el trabajo en equipo que requiere el curso de la "Guía Integrada de Investigación". Luego hay que hacerse presente en las

\section{(C) $(0 \odot$}

La Revista Estudios es editada por la Universidad de Costa Rica y se distribuye bajo una Licencia Creative Commons Atribución-NoComercial-CompartirIgual 3.0 Costa Rica. Para más información envíe un mensaje a 
Especial: Profesores de Estudios Generales investigan

instancias de decisión, para que las cosas cambien. Autocrítica y no complacencia.

Todo esto debe hacerse porque después de más de cincuenta años de existencia de este curso, el valor de la investigación sigue estando vigente, es pertinente desde el punto de vista institucional y desde el nacional, pues probablemente hoy más que nunca se necesita la investigación para aportar pensamiento crítico y cambio en la comunidad nacional. Es pertinente por cuanto la normativa lo exige, es pertinente porque se ha heredado un visón de formación humanista que se tiene la responsabilidad de plenificar y desarrollar en un contexto contemporáneo cada vez más deshumanizado. Pero nada de esto será posible sin autocrítica y acciones concretas de cambio que la acompañen.

\section{Bibliografía}

Azofeifa, I. F. (1979). Humanismo Crítico. Los polémicos Estudios Generales. San José: EUCR.

Barahona-Jiménez, L. (2015) La Universidad de Costa Rica: (1940-1973). 2da.edic. San José: Editorial de la Universidad de Costa Rica.

Bonilla, A. (1969). Objetivos de los Estudios Generales. San José: EUCR.

Carvajal-Alvarado, G. (2005). Los Estudios Generales en la Universidad de Costa Rica. San José: Editorial Librería Alma Mater.

Chaverri, A., González, A. y Pino, G. (1994). Sobre el concepto de Guía Académica. Revista Estudios. Número 11, Costa Rica.

Centro de Investigaciones Históricas (1991) Historia de la educación superior en Costa Rica. San José: Oficina de Publicaciones de la Universidad de Costa Rica.

Chinchilla-Gutiérrez, S. (2011). Estudios Generales en sus hechos. San José: Editorama.

\section{(c) (i) (9) (2)}

La Revista Estudios es editada por la Universidad de Costa Rica y se distribuye bajo una Licencia Creative Commons Atribución-NoComercial-CompartirIgual 3.0 Costa Rica. Para más información envíe un mensaje a 
Especial: Profesores de Estudios Generales investigan

Constitución Política de la República de Costa Rica (2019) Recuperado de http://www.asamblea.go.cr/Documents/Constitucion_Politica.pdf

Escuela de Estudios Generales. (1991). El Cincuentenario de la Universidad de Costa Rica y la Revolución Científica y Tecnológica. El Humanismo Contemporáneo en los Estudios Generales. Revista Estudios. Número 9, 1991. San José: Sección de Historia de la Cultura, Escuela de Estudios Generales, Universidad de Costa Rica.

Escuela de Estudios Generales. (2003) Memoria Histórica del Congreso de Humanidades: "Cultura, Universidad y Humanismo en el Siglo XXI". Número Extraordinario. Abril 2003. Girasol: Revista de la Escuela de Estudios Generales. Universidad de Costa Rica. San José: Editorial de la Universidad de Costa Rica.

Esquivel-Estrada N. H.( 2003). ¿Por qué y para qué la formación humanista en el educación superior?. Revista Ciencia Ergo Sum. Noviembre 2003-Febrero 2004, año/vol. 10, número 003. México: Universidad Autónoma del Estado de México. Pp.309-320 Recuperado de: https://www.redalyc.org [29-09-19]

González-Villalobos, P. (1989). La Universidad de Santo Tomás. San José: Editorial de la Universidad de Costa Rica.

Karpinsky, R. M. (1982). Estudios Generales: Concepción Teórica y Praxis Académica. San Pedro: UCR.

Láscaris-Conmeno, C. (1958). Teoría de los Estudios Generales. San Pedro: UCR. Rivero-Franyutti, A. (2013). ¿Qué son hoy las humanidades y cuál ha sido su valor en la universidad?. Revista de la educación superior, 42(167), 81-100. Recuperado de http://www.scielo.org.mx/scielo.php?script=sci_arttext\&pid=S0185$27602013000300003 \& \operatorname{lng}=$ es\&tlng=es.

Soto-Valverde, G.A. (2007). 50 aniversario de los Estudios Generales de la Universidad de Costa Rica: Documentos fundamentales. San José: Sección de Impresión del SIEDIN

Universidad de Costa Rica. Estatuto Orgánico. San Pedro: UCR. Sus actualizaciones en la página electrónica de la Universidad de Costa Rica: Consejo Universitario: Normativa Universitaria. Recuperado de http://www.cu.ucr.ac.cr/estatuto-organico.html

\section{(C) $(00$}

La Revista Estudios es editada por la Universidad de Costa Rica y se distribuye bajo una Licencia Creative Commons Atribución-NoComercial-CompartirIgual 3.0 Costa Rica. Para más información envíe un mensaje a 